\title{
Takayasu Arteritis as a Secondary Cause of Arterial Hypertension
}

\author{
Pedro Manuel Oliveira, ${ }^{(1)}$ Paula Fereira, ${ }^{(1)}$ Fábio Murteira, ${ }^{(1)}$ Inês Rueff Rato, ${ }^{(1)}$ Marta Barbedo
}

Centro Hospitalar de Vila Nova de Gaia, Vila Nova de Gaia - Portugal

\begin{abstract}
Takayasu arteritis (TA) is a rare type of vasculitis that affects mainly the aorta and its major branches. It is highly similar to giant cell arteritis (GCA), and differentiation between them may not be achieved even by histological examination. Arterial hypertension is typical of TA and is caused by stenosis of the renal arteries. Here we report the case of a 59-year-old woman, with a history of dyslipidemia and anemia, seen in the Internal Medicine department for resistant hypertension. Evaluation of secondary causes led to stenosis of the renal arteries. Assessment of target organ involvement was performed by computed tomography angiograph which revealed ectasia of the aortic arch and ascending aorta, tortuous course of the brachiocephalic trunk and the proximal portion of the right common carotid artery; positron-emission tomography which showed diffuse increased uptake in the ascending aorta, compatible with large vessels vasculitis. The patient was submitted to aortic valve replacement with a biological prosthesis combined with myocardial revascularization (BentallDe Bono procedure). Aortic biopsy specimens showed anatomical and pathological features of GCA and TA. Due to persistently uncontrolled hypertension, prednisone $60 \mathrm{mg}$ was initiated,with significant improvement in patient's condition.
\end{abstract}

\section{Introduction}

Takayasu arteritis (TA) is a type of vasculitis that affects mainly the aorta and its major branches, but coronary and pulmonary arteries may also be involved. ${ }^{1}$ It is more

\section{Keywords}

Takayasu Arteritis; Hypertension; Renal Artery Obstruction; Aorta, Thoracic; Computed Tomography Angiography/methods. common in women (80-90\%) than men, particularly in women aged between 10 and 40 years., ${ }^{2,3}$ Although several cases have been reported worldwide, with an incidence of 2.6 cases per million per year, TA has been more extensively studied in Japan. ${ }^{1}$

Clinical manifestations of TA consist of signs and symptoms of systemic and vascular inflammation, including constitutional symptoms, hypertension, arthralgia, carotidynia, blood pressure difference between the upper limbs, angina, and neurological symptoms. ${ }^{4}$ Hypertension is observed in almost half of patients ${ }^{4}$ due to stenosis of renal artery or reduced aortic elasticity and it is only present in TA. 5,6

The diagnosis of TA is usually made based on the Ishikawa ${ }^{7}$ or the American College of Rheumatology criteria. ${ }^{3}$ TA share similar characteristics and may be confounded with giant cell arteritis (GCA), and even histological analysis does not allow differentiation between these conditions. ${ }^{8}$

The treatment of TA consists of corticosteroids (with or without corticosteroid-sparing agent), and surgical intervention may be necessary in cases of irreversible arterial stenosis and giant aneurysms. ${ }^{9}$ Both anemia and sedimentation rate usually improve, ${ }^{5}$ and, more rarely, arterial stenosis is reversed with immunosuppression. ${ }^{9}$ However, progressive worsening of the lesions may be common.

\section{Case Report}

Here we report the case of a 59-year-old Caucasian woman, with a history of hypertension (under pharmacological treatment since the age of 18), dyslipidemia, obstructive sleep apnea with use of continuous positive airway pressure, thyroid nodules, chronic disease anemia, and recent episode of lacunar stroke and partially recovered left hemiparesis.

Mailing Address: Pedro Oliveira

Rua Conceição Fernandes, s/s. Postal Code: 4400-129, Vila Nova de Gaia - Portugal.

E-mail: pedromvaoliveira@gmail.com 
The patient was referred to the internal medicine department because of resistant hypertension. Physical examination revealed a pulsatile mass in the right neck, and no other abnormal findings or complaints. The patient underwent ambulatory blood pressure monitoring, which revealed mean blood pressure of $150 / 70 \mathrm{mmHg}$ despite the use of three medications (lisinopril, hydrochlorothiazide, amlodipine) and correct adherence to the treatment.

Evaluation of secondary causes - thyroid function, aldosterone-to-renin, and determination of metanephrine, vanillylmandelic acid, and urinary cortisol levels did not show any abnormalities. Doppler study of the renal arteries demonstrated a zone of flow acceleration within the proximal renal artery bilaterally, suggestive of stenosis. Computed tomography angiography was performed, which confirmed narrowing of the initial segment of the right renal artery, without dilation of the distal segment, raising the possibility of ostial stenosis (Figure 1).
In addition to stroke, the echocardiogram revealed left ventricular hypertrophy, moderate aortic insufficiency, and mild mitral valve insufficiency. Microalbuminuria was not detected. Carotid Doppler showed diffuse bilateral thickening of the intima-media complex, small plaques in the left bifurcation and stenosis $<10 \%$, and involvement of small vessels downstream in both carotid arteries.

Due to uncontrolled hypertension despite therapy optimization and results of the carotid Doppler ultrasound, the patient was referred for computed angiography of the chest and neck, which revealed ectasia of the aortic arch and ascending aorta (largest diameter: $5.5 \mathrm{~cm}$ ), associated with a tortuous course of the brachiocephalic trunk and the proximal portion of the right common carotid artery (Figure 2). The patient also underwent cardiac catheterization which showed stenosis $(20 \%)$ of distal common trunk, irregular descending artery, stenosis ( $85 \%$ ) of the proximal circumflex artery and stenosis in two segments (90\% and $50 \%$ ) of the medial third of the right coronary artery.

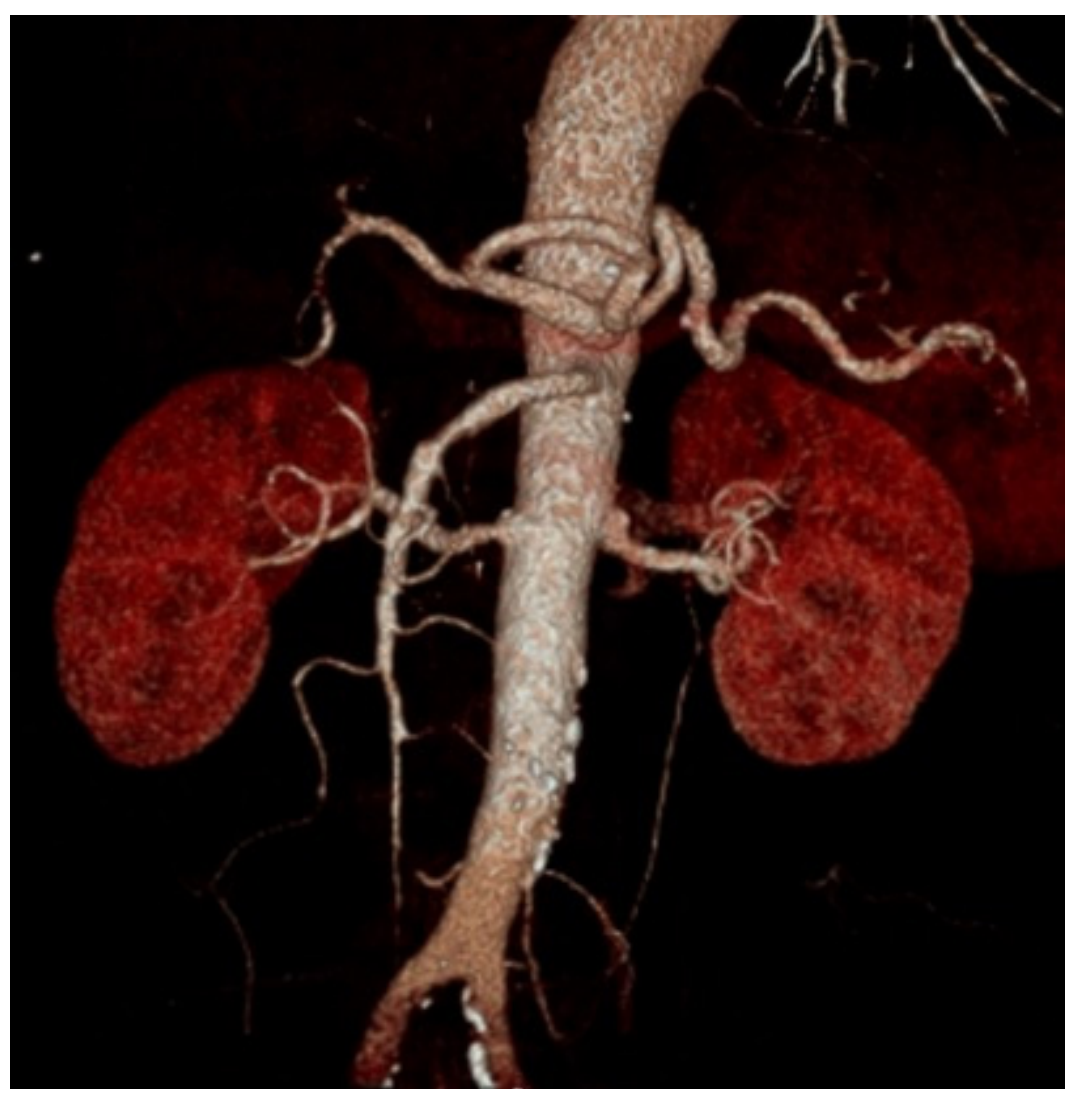

Figure 1 - Contrast-enhanced computed tomography showing narrowing of the right renal artery proximal segment 


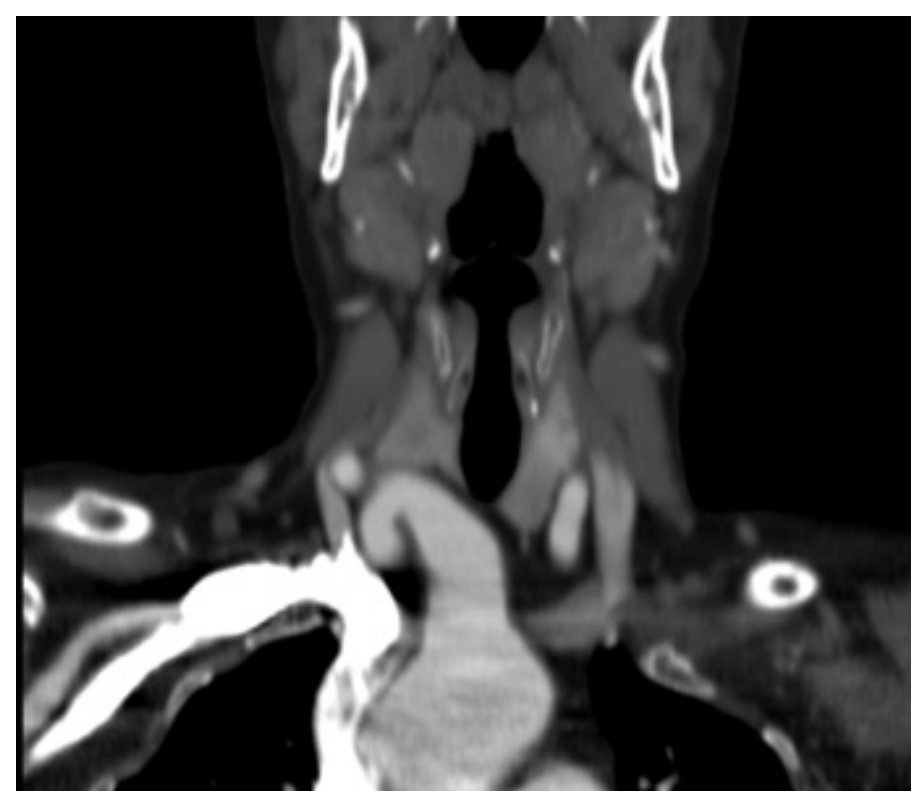

Figure 2 - Contrast-enhanced computed tomography of the neck showing ectasia of the aortic arch

Considering the vascular changes (renal, aortic, carotid, and coronary arteries) detected and the history of stroke, the hypothesis of TA was raised. Except for an erythrocyte sedimentation rate in the first hour of $59 \mathrm{~mm} / \mathrm{h}$, no other abnormalities were found in the immunological evaluation. Positron emission tomography showed diffuse increased uptake of F18-fluorodeoxyglucos (FDG) in ascending aorta and proximal aortic arch, and functional changes compatible with an inflammatory process namely large vessel vasculitis.

Due to the presence of ascending aortic aneurysm, the case was discussed by a multidisciplinary team including the cardiothoracic surgery team, who decided to perform aortic valve replacement with a biological prosthesis combined with myocardial revascularization (Bentall-De Bono procedure) (Figure 3). During surgery, aortic biopsy specimens were obtained which showed anatomical and pathological features of GCA or TA. No post-operative complications were noticed. One month later, the patient still had uncontrolled hypertension $(150 / 80 \mathrm{mmHg})$ and episodes of heart failure decompensation. Corticosteroid therapy was then initiated, with prednisone $60 \mathrm{mg}$ (1 mg/Kg/day). The patient experienced significant improvement of complaints and blood pressure control $(110 / 60 \mathrm{mHg})$, reduction of inflammatory parameters (sedimentation rate $14 \mathrm{~mm} / \mathrm{h}$ ), and resolution of anemia and inflammatory disease. Treatment with prednisone
$60 \mathrm{mg}$ was continued for two months, followed by a gradual weaning during the subsequent two months, until complete withdrawal. The patient has been well for two years, without immunosuppression, with controlled hypertension and no complaints.

\section{Discussion}

TA is a rare condition in Western countries. ${ }^{10}$ Its diagnosis is based on clinical and imaging criteria, since aortic biopsy is not always possible. Contrastenhanced computed tomography (or, alternatively, magnetic resonance) is essential for evaluation of the arterial lumen. ${ }^{4}$ Markers of acute inflammation (C-reactive protein and sedimentation rate) are used to monitor therapy response, ${ }^{9}$ and may be normal even in active disease. ${ }^{10}$

The "diagnostic path" in the current case deserves attention. This hypertensive patient was referred for exclusion of secondary hypertension, as she had elevated blood pressure despite receiving triple therapy, and frequent episodes of hypertensive urgencies. In addition, the patient had a history of stroke at a relatively young age, which reinforced the importance of controlling blood pressure levels, and presence of dyspnea and pulsatile neck mass at physical examination related to current disease. Evaluation of secondary causes and target organs led to important 


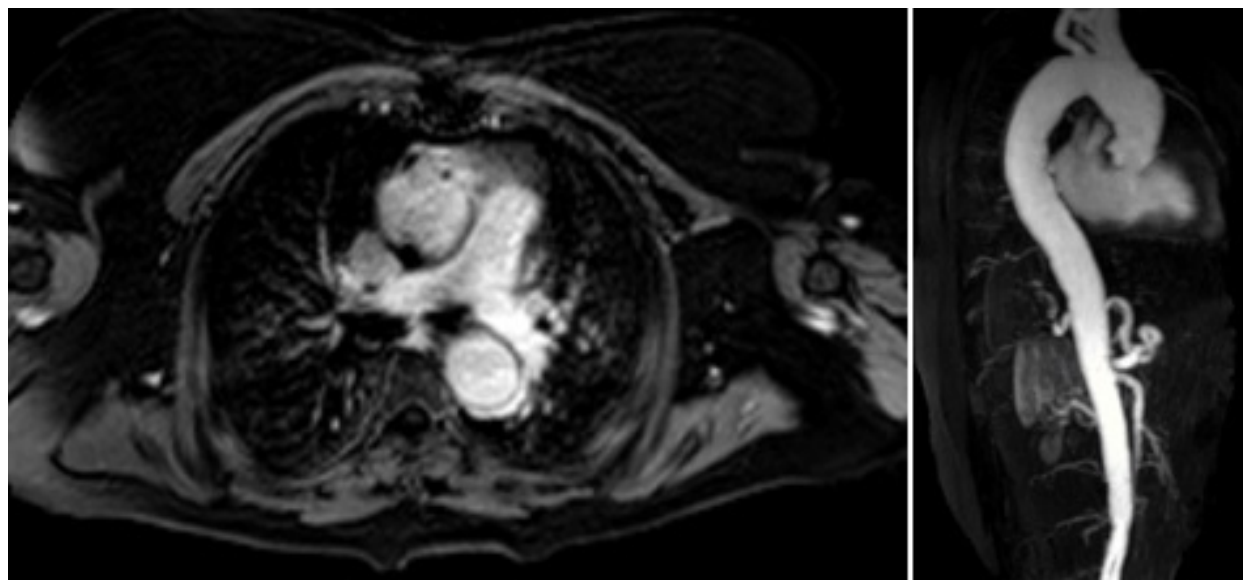

Figure 3 - Post-operative magnetic resonance showing correct placement of the biological prosthesis, without complications

findings - ostial stenosis of the right renal artery and diffuse thickening of carotid walls. These findings raised the suspicion of TA, and the next step was the angiographic study of neck and chest, which revealed ectasia of the aortic arch and ascending aorta. The increased sedimentation rate reinforced the hypothesis of TA, which was practically confirmed by positron emission tomography. The patient then underwent surgical repair of ectasia and the histological analysis of biopsy specimens confirmed the diagnosis. It is worth mentioning that the diagnosis is rarely made by histological examination, as it is difficult to be performed. ${ }^{4}$ The improvement of symptoms, with normalization of blood pressure and resolution of anemia, was achieved by corticosteroid therapy.

Although TA and GCA are very similar and easily confused conditions, some features allow to distinguish them (Table 1$)^{4}$. For example, while GCA commonly affect women over 50 years of age, TA is more common among women younger than 40 years old. ${ }^{8}$ However, emerging evidence indicates that both TA and GCA represent the same disease, ${ }^{11}$ as they have several overlapping clinical features.

In this case report, we present a case of TA in a 50 -year-old woman. Both patient age and ethnicity would lead to the hypothesis of GCA, but stenotic lesions of the renal arteries associated with the hypertension status led to a definite diagnosis of TA, since this is not a common feature of GCA. ${ }^{5,6}$ Several vascular beds were affected in this patient - the aorta, heart and brain, and the involvement of the aorta and main branches also reinforce the diagnostic suspicion of TA.

Corticosteroids seem to be effective in relieving symptoms in both diseases. Histopathological study does not allow distinguishing between GCA and TA, due to the similar features between them.

The treatment of TA, as previously described, is based on systemic corticosteroid therapy. ${ }^{9}$ However, considering that recurrence is common and the longterm use of corticosteroid may lead to important side effects, other immunosuppressive agents may be used, including methotrexate (20-25 mg once a week) or azathioprine $(2 \mathrm{mg} / \mathrm{Kg} /$ day $) .{ }^{9}$ Some situations require surgical intervention, such as hypertension with critical stenosis of the renal artery, claudication of extremities that negatively affect the performance of daily activities, cerebral ischemia, severe stenosis of three or more cerebral vessels, moderate aortic insufficiency or cardiac ischemia with confirmed coronary disease. ${ }^{12}$ This was precisely what was performed in this case reported - the patient was submitted to aortic prosthesis implantation and coronary revascularization, since aortic insufficiency and cardiac ischemia were detected by catheterization. However, since stenosis of the renal artery was not critical, a surgical intervention in this artery was postponed. Immunosuppression with systemic corticosteroid was then initiated. This was the correct therapeutic decision, since this approach led to control of blood pressure without the need of additional intervention. 
Table 1 - Differentiation between giant cell arteritis and Takayasu arteritis, adapted from UptoDate ${ }^{4}$

\begin{tabular}{lll} 
& Giant cell arteritis & Takayasu arteritis \\
\hline Female : male ratio & $3: 2$ & $7: 1$ \\
\hline Age of onset & $>50$ years & $<40$ years \\
\hline Arterial hypertension & Rare & Common \\
\hline Ethnicity (predominance) & European & Asian \\
\hline Vascular beds commonly affected & External carotid artery branches & Aorta and its main branches \\
\hline Response to corticosteroids & Excellent & Common \\
\hline Surgery required & Rare & \\
\hline Histopathology & Granulomatous inflammation (like) & \\
\hline
\end{tabular}

TA is a rare disease, with heterogeneous clinical presentation. Thus, its diagnosis may be difficult and require a high degree of suspicion. A delay in the prognosis, and consequently in treatment initiation worsens the prognosis and increases the possibility of complications. ${ }^{10}$ TA has a high morbidity; $74 \%$ of patients refer a significant impact on daily activities, and $23 \%$ of patients refer loss of work productivity. ${ }^{10}$ Important sequelae of the disease include brain ischemia, rapidly growing aneurysms, pulmonary hypertension, aortic rupture, aortic valve insufficiency, and acute myocardial infarction. ${ }^{6}$

\section{Conclusion}

The case here reported illustrates how a correct clinical history-taking, a complete physical examination, and carefully selected complementary diagnostic tests can lead to a correct diagnosis. In fact, the concomitant presence of difficult-to-control hypertension, anemia, stroke, pulsatile neck mass, stenosis of the renal artery and carotid Doppler ultrasound findings raised the suspicion of TA. TA is a rare and potentially severe disease whose diagnosis depends on a high degree of suspicion and physician's alertness.

\section{Author contributions}

Conception and design of the research: Oliveira PM. Acquisition of data: Oliveira PM. Writing of the manuscript: Oliveira PM, Ferreira P, Murteira F, Rato IR. Critical revision of the manuscript for intellectual content: Barbedo M.

\section{Potential Conflict of Interest}

No potential conflict of interest relevant to this article was reported.

\section{Sources of Funding}

There were no external funding sources for this study.

\section{Study Association}

This study is not associated with any thesis or dissertation work: Pedro Manuel Oliveira, Paula Ferreira P, Fábio Murteira F, Inês Rueff Rato IR, Marta Barbedo M.

\section{Ethics approval and consent to participate}

This article does not contain any studies with human participants or animals performed by any of the authors. 


\section{References}

1. Manfrini, $\mathrm{O}$, Bugiardini, R. Takayasu's arteritis: A case report and a brief review of the literature. Heart Int. 2006;2(1):66-71. Doi:10.4081/hi.2006.66

2. Lupi-Herrera E, Sánchez-Torres G, Marcushamer J, Mispireta J, Horwitz S, Espino Vela J. Takayasu's arteritis. Clinical study of 107 cases. Am Heart J.1997;93(1):94-103. Doi:10.1016/s0002-8703(77)80178-6.

3. Arend WP, Michel BA, Bloch DA, Hunder GG, Calabrese LH, Edworthy SM, et al. The American College of Rheumatology 1990 criteria for the classification of Takayasu arteritis. Arthritis Rheum.2010;33(8):1129-34. doi:10.1002/art.1780330811.

4. Merkel, PA. Clinical features and diagnosis of Takayasu arteritis. UpToDate, 2019.

5. Kerr GS. Takayasu's Arteritis. Rheum Dis Clin North Am, 1995 Nov;21(4):1041-58

6. Docken, WP. Diagnosis of giant cell arteritis, UpToDate, 2019.
7. Ishikawa K. Diagnostic approach and proposed criteria for the clinical diagnosis of Takayasu's' arteriopathy. J Am Coll Cardiol. 1988;12:964-72. doi:10.1016/0735-1097(88)90462-7.

8. Michel BA, Arend WP, Hunder GG. Clinical differentiation between giant cell (temporal) arteritis and Takayasu's arteritis. J Rheumatol. 1996 Jan;23(1):106-11.

9. Merkel, PA. Treatment of Takayasu arteritis, UpToDate, 2020.

10. Mason JC. Takayasu arteritis - advances in diagnosis and management. Nature Rev Rheumatol.2010;6(7):406-15. doi:10.1038/nrrheum.2010.82

11. Maksimowicz-McKinnon K, Clark TM, Hoffman GS. Takayasu Arteritis and Giant Cell Arteritis. Medicine.2009;88(4):221-6. doi:10.1097/ MD.0b013e3181af70c1.

12. Johnston SL, Lock RJ, Gompels MM. Takayasu arteritis: a review. J Clin Pathol. 2002 Jul;55(7):481-6. Doi:10.1136/jcp.55.7.481. 